\title{
BARYONS AS SOLITONS IN EFFECTIVE CHIRAL FIELD THEORIES ${ }^{1}$
}

\author{
G. HOLZWARTH ${ }^{2}$ \\ Fachbereich Physik, Universit $\Xi$-GH-Siegen \\ 57068 Siegen, Germany
}

\begin{abstract}
This lecture comprises some recent developments concerning the description of baryons as topological solitons in effective chiral meson theories.

In the first part one-loop corrections to the classical tree approximation are discussed. This involves renormalization of low-energy coupling constants and evaluation of the finite next-to-leading-order terms in the $1 / N_{c}$ expansion. In contrast to the corresponding procedure in the meson sector the magnitude of the chiral gradients involved in the soliton profile requires that counter terms and finite loop contributions be calculated to all chiral orders. Recent results for various nucleon observables are presented. They show that the $1 / N_{c}$ expansion essentially works as expected.

In the second part electro-magnetic nucleon form factors (FFs) with relativistic corrections are evaluated in a chiral soliton model including vector mesons. The magnetic FF $G_{M}^{p}$ is shown to agree well with new SLAC data for spacelike $Q^{2}$ up to $30(\mathrm{GeV} / \mathrm{c})^{2}$ if superconvergence is enforced. The electric $\mathrm{FF} G_{E}^{p}$ is dominated by a zero in the few $(\mathrm{GeV} / \mathrm{c})^{2}$ region due to a low-lying zero in the non-relativistic electric FF in tree approximation.

The third part describes how to extract the strong $\pi N N$ form factor from chiral soliton models, taking due care of the local metric created by the presence of the soliton. When used in a one-boson-exchange model for the nucleon-nucleon (NN) interaction, deuteron properties and phase parameters of NN scattering are reproduced as well as in conventional NN models that apply a hard monopole form factor at the $\pi N N$ vertex.
\end{abstract}

hep-ph/9511249

November, 1995

${ }^{1}$ Lecture presented at the International School of Nuclear Physics, 17th Course:" Quarks in Hadrons and Nuclei" at the Ettore Majorana Centre for Scientific Culture, Erice, Italy, September 19th-27th, 1995. 


\section{INTRODUCTION}

Topological soliton models for structure and dynamics of baryons are based on effective nonlinear lagrangians for selected mesonic degrees of freedom. These usually comprise the set $U(\boldsymbol{x}, t)$ of pseudoscalar Goldstone bosons of spontaneously broken chiral symmetry, but also the light vector meson octet, and axial vector mesons have been included.

Chiral Perturbation Theory (ChPT) [1,2] provides a systematic way to establish order by order the effective lagrangian $\mathcal{L}(U)$ for the pseudoscalar chiral Goldstone bosons. To the extent that the relevant coupling constants can be fixed with sufficient accuracy for a consistent description of low-energy mesonic processes, the resulting part of the effective lagrangian ideally should coincide with the chiral lagrangian underlying the soliton model for baryons. This must be so because far away from the center of a soliton the fluctuations of the chiral field describe mesons which should interact in the same way as the mesons in the vacuum sector.

It is always the first step in the actual use of a chiral effective lagrangian to construct a static solution $U_{0}(\boldsymbol{x})$ which minimizes the classical effective action. Depending on boundary conditions, chiral lagrangians allow for topologically distinct sets of classical solutions, characterized by suitable integer winding numbers $n$. Following Skyrme [3] and Witten [4], the identification of the topological index $n$ with baryon number $B$, establishes a close link between the concepts of ChPT and soliton models for baryons.

Although it is quite remarkable that the presently known low-energy coupling constants of ChPT support stable static soliton solutions, there still are crucial differences in the practical application of both concepts:

In ChPT the scale of external momenta involved in a physical process limits the chiral order $i_{\text {max }}$ to which the effective lagrangian $\mathcal{L}$ has to be considered:

$$
\mathcal{L}(U)=\int \sum_{i=2,4,6, . .}^{i=i_{\max }} c_{i}\left(L_{\mu}\right)^{i} d^{3} x
$$

where $L_{\mu}$ denotes the chiral gradients

$$
L_{\mu}=U^{\dagger} \partial_{\mu} U
$$

For a soliton solution in the $B=1$ sector the gradients of the soliton profile are of the order of $(\pi / d)$ where $d$ is the typical size of the soliton. For $d \sim 1 \mathrm{fm}$ we have $\pi / d \sim 700 \mathrm{MeV}$, i.e. the gradients typically are of the order of the $\rho$-mass $m_{\rho}$. This excludes a justification based on the power of chiral gradients, for truncating the effective lagrangian to some low chiral order. The limitation to low powers of $L_{\mu}$ in $\mathcal{L}$ which is necessary for practical reasons, then implies the assumption that the renormalized coupling constants $c_{i}$ for $i>i_{\max }$ are so small that effects of the omitted higher-order terms may be safely neglected.

Numerous investigations of the (chiral-order-four) Skyrme model and higher-order extensions have shown, that chiral profile and baryonic properties in tree approximation do not differ drastically, if only the size of the soliton is of comparable magnitude. In fact, to some extent this holds even for chiral lagrangians with vector mesons included, which effectively sum up terms to arbitrarily high chiral orders [5].

Going beyond the classical tree approximation requires evaluation of the chiral field fluctuations $\pi(\boldsymbol{x}, t)$ around the static soliton $U_{0}(\boldsymbol{x})$

$$
U(\boldsymbol{x}, t)=U_{0}(\boldsymbol{x}) \exp \left[i \pi(\boldsymbol{x}, t) / f_{\pi}\right] .
$$


which underlies the dynamics of these fluctuations be consistent with the equation of motion which determines the static soliton itself. (Otherwise zero modes will no longer be zero modes, terms linear in the fluctuational fields will not vanish, and the meson-soliton interaction $V$ will depend on the choice of the parametrization chosen for the fluctuational field. ) Therefore in $h_{S}^{2}$ all terms have to be kept which arise from the full effective lagrangian used in tree approximation to evaluate the classical soliton.

Renormalization of loop corrections [9 [1] then requires counter terms which already in one-loop approximation renormalize the parameters of all higher chiral orders in the effective lagrangian $\mathcal{L}$. Still, as stated for the meson sector in Weinberg's power-counting rule [1], the renormalized coupling constants for the terms of some given chiral order $i$ pick up loop contributions only from those terms in $\mathcal{L}$ with chiral order $i-2$ and less. This allows for the important fact that renormalization in the meson sector simultaneously renormalizes also the soliton sector. However, the resulting finite loop-corrections to physical quantities involve all powers of the terms kept in the lagrangian and cannot be truncated because the gradients involved are not small. This is an important difference to the procedures applied in the meson sector and it rules out early attempts [13] to calculate loop corrections in the soliton sector by counting chiral orders in the same way as in the meson sector.

So, to restate the essentials: i) The renormalization of loops in the soliton sector leads to the same renormalized coupling constants as in the meson sector. ii) The finite loop contributions to the observables of interest contain all powers of the terms which are kept in the effective lagrangian, i.e. they comprise all chiral orders. iii) A truncation of the effective lagrangian can not be justified on grounds of increasing chiral order, but it implies the assumption that the renormalized coupling constants for the omitted terms are very small. (Such a statement of course is scale dependent).

From the foregoing it is quite evident that using the established parts of the effective chiral lagrangian in the soliton sector lacks the rigor of the systematic ChPT scheme, while going beyond the established parts turns this fundamental tool into a mere QCD-inspired model for baryon structure and dynamics. While in ChPT loop corrections are in tight correspondence with higher orders in the chiral expansion, in the soliton sector tree, one-loop, two-loop approximation etc. merely represent the first terms in the $1 / N_{c}$ expansion, with each term comprising all chiral orders, independent of the maximal chiral order $i_{\max }$ kept in the effective action. Thus, evaluation of one-loop corrections in the soliton sector provides information not only about the magnitude of these $1 / N_{c^{-}}$corrections, but an eventually remaining scale dependence of physical observables indicates how seriously the lack of rigor may affect the results.

\section{AN EXAMPLE: THE NUCLEON MASS}

As an example let us consider the nucleon mass in more detail. In an effective field theory characterized by a classical (Euclidean) action $S^{E}$ the mass of the lowest quantum eigenstate is given by [12]

$$
M=-\lim _{\tau \rightarrow \infty} \frac{1}{\tau} S^{E}
$$

where $\tau$ limits the (imaginary-)time integral in the action $S^{E}$. Tree and one-loop approximation are the leading and next-to-leading-order terms in a $1 / N_{c}$ expansion of the action

$$
S^{E}=S_{0}^{E}-\frac{1}{2} \operatorname{Tr} \ln D^{E}+\cdots
$$

where $S_{0}^{E}$ is the action taken at the static classical solution and $D^{E}$ is the operator which determines the harmonic fluctuations around the classical minimum. For chiral lagrangians the classical static configuration is the hedgehog soliton while the fluctuations asymptotically correspond to the pseudoscalar mesons. The scale in the meson-soliton interaction is set by gradients of the soliton profile which as we discussed above are of the order of of the $\rho$-mass $m_{\rho}$. Generally, therefore, it is not sufficient to evaluate 
of chiral derivatives which occur in the effective lagrangian. Similarly, it is not possible to obtain the zero modes in Born approximation; however, they provide the most important part of the quantum corrections to the baryon mass.

After performing the time integral in $S^{E}$ the baryon mass then is given as

$$
M=M_{S}+E_{\text {cas }}
$$

with $\mathcal{O}\left(N_{c}\right)$ classical soliton mass $M_{S}$ and $\mathcal{O}(1)$ Casimir energy

$$
E_{c a s}=\frac{1}{2}\left(\operatorname{tr} h_{S}-\operatorname{tr} h_{\infty}\right)
$$

The hamiltonian $h_{S}$ governs the dynamics of the soliton fluctuations. Asymptotically, far away from the soliton, $h_{S}^{2}$ turns into the kinetic energy operator $h_{\infty}^{2}=\boldsymbol{p}^{2}+m_{\pi}^{2}$ for free pions. In the Casimir energy the infinite $\mathcal{O}(1)$ vacuum energy contribution is subtracted.

The Casimir energy is a formal object which requires renormalization. In order to determine the relevant counter terms (and only for that purpose) we separate $h_{S}^{2}$ into kinetic part $h_{0}^{2}$ and chiral covariant momentum-independent interaction part $V$

$$
h_{S}^{2}=h_{0}^{2}+V
$$

and consider the formal Taylor expansion of the square root of $h_{S}^{2}$ in (7) in powers of the interaction $V$

$$
\operatorname{tr} h_{S}=\operatorname{tr} h_{0} \sum_{k=0}^{\infty} \lambda_{k}\left(\frac{V}{h_{0}^{2}}\right)^{k}, \quad\left(\lambda_{0}=1, \quad \lambda_{1}=1 / 2, \quad \lambda_{2}=-1 / 8, \ldots\right)
$$

The ultraviolet divergencies reside (for $3+1$ dimensions) in the first three terms $k=0,1,2$, while terms with $k>2$ are finite. The series may be visualized diagrammatically as a sum of one-pion-loops with $k$ insertions of the pion-soliton vertex $V$ :

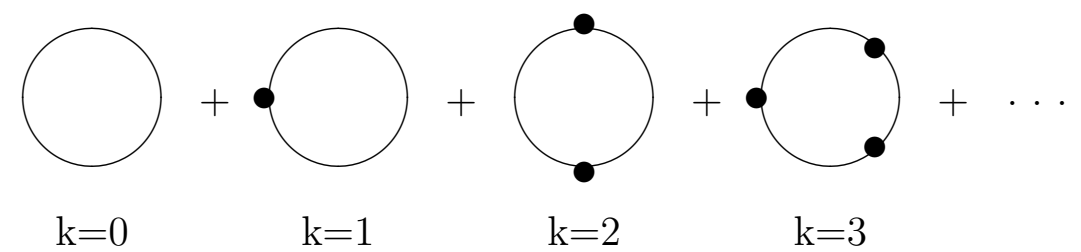

Fig. 1. Graphical representation of the one-loop multi-vertex series (9).

There is no small expansion parameter in this series and as we have stressed before, it must be summed to all orders. Formally, the renormalization procedure consists of the following: Subtract from $E_{\text {cas }}$ counterterms which remove the infinities in the series (9) and add them again:

$$
E_{\text {cas }}=\frac{1}{2} \operatorname{tr}\left(h_{S}-h_{0}-\frac{1}{2 h_{0}} V+\frac{1}{8 h_{0}^{3}} V^{2}\right)+\frac{1}{2}\left(\alpha_{0} a_{0}+\alpha_{1} a_{1}+\alpha_{2} a_{2}\right)
$$

with the divergent integrals

$$
\alpha_{k}=\lambda_{k} /(2 \pi)^{3} \int d^{3} p\left(\boldsymbol{p}^{2}+m_{\pi}^{2}\right)^{1 / 2-k}
$$

which are conveniently handled in dimensional regularization with renormalization scale $\mu$. In the $(\bar{M} S)$ subtraction scheme the infinite parts of the integrals $\alpha_{k}$ are absorbed into scale-dependent renormalized coupling constants $c_{i}(\mu)$ in the (sufficiently general) effective lagrangian (this is always possible because the spatial integrals $a_{0}, a_{1}, a_{2}$ are chirally covariant). Remaining scale-dependent finite parts of the $\alpha_{k}$ integrals, together with the scale-independent finite part of $E_{c a s}$ in (10) then constitute the scaledependent finite one-loop correction $E_{\text {cas }}(\mu)$ to the classical soliton mass $M_{S}(\mu)$ (which is implicitly scale dependent via the renormalized coupling constants $\left.c_{i}(\mu)\right)$.

Exact evaluation of $\operatorname{tr} h_{S}$ requires knowledge of the exact scattering eigenstates for $h_{S}^{2}$. They have been 


\section{SOME RESULTS AND REMARKS ABOUT THE $1 / N_{C}$-EXPANSION}

At present results for one-loop corrections have only been obtained in the framework of chiral $\mathrm{SU}(2) \times \mathrm{SU}(2)$, i.e. for purely pionic dynamics. In this case the chiral-order-four part of $\mathcal{L}$ consists (apart from small terms proportional to the pion mass) of only two terms, the antisymmetric Skyrme term

$$
\mathcal{L}_{A}^{(4)}=\frac{1}{32 e^{2}} \int \operatorname{tr}\left[L_{\mu}, L_{\nu}\right]^{2} d^{3} x
$$

and the symmetric term

$$
\mathcal{L}_{S}^{(4)}=\frac{\gamma}{8 e^{2}} \int\left(\operatorname{tr} L_{\mu} L_{\mu}\right)^{2} d^{3} x
$$

in addition to the usual (chiral-order-two) part

$$
\mathcal{L}^{(2)}=\frac{f_{\pi}^{2}}{4} \int\left(-\operatorname{tr} L_{\mu} L^{\mu}+m_{\pi}^{2} \operatorname{tr}\left(U+U^{\dagger}-2\right)\right) d^{3} x .
$$

In ChPT, with the conventional choice for the renormalization scale $\mu=m_{\rho}$, the magnitude of the Skyrme parameter $e$ has been determined to lie in the range $5.8<e<7.2$, while $\gamma$ is compatible with zero. As we have remarked already, this order-four lagrangian stabilizes static soliton solutions. However, for a reasonable size of the resulting baryon, values of the Skyrme parameter around $e \approx 4$ are necessary. This is a clear indication that for a reliable description of baryons in the soliton sector more terms in the chiral expansion of $\mathcal{L}$ are required. There are, however, so many possible types of terms all with unknown coupling constants, that presently one reasonable strategy seems to be restriction to $\mathcal{L}^{(4)}$ with a choice for $e$ which differs from its value determined in ChPT, but effectively represents the influence of the higher-chiral-order terms on the soliton solutions. A posteriori, this compromise may be justifiable if it turns out that the one-loop corrections lead to significant improvement for many baryonic observables and, as we have discussed earlier, that the results show only a mild dependence on the renormalization scale $\mu$.

Fig. 2 shows results obtained for the baryon mass with $e=4.25$ and $\gamma=0$ at $\mu=m_{\rho}$. Through the scale dependence of the renormalized coupling constants the classical soliton mass $M_{S}(\mu)$ varies between 1400 and $1800 \mathrm{MeV}$ in the scale range of $450 \mathrm{MeV}<\mu<1200 \mathrm{MeV}$. Remarkably, the one-loop correction compensates this dependence to such a degree that in the scale range from the $\eta$-mass $m_{\eta}$ up to $1 \mathrm{GeV}$ the resulting baryon mass is constant near $946 \mathrm{MeV}$ with very good accuracy. Below $\mu \approx 500 \mathrm{MeV}$ the value of $\gamma(\mu)$ approaches the limit where the symmetric term (13) destabilizes the classical soliton solution.

Similarly, numerical results can be obtained for other observables by recalculating tree and one-loop contributions to the mass in presence of suitably chosen external fields. Evaluating derivatives of the baryon mass with respect to the strengths of the imposed external fields provides magnitude and scale dependence for any desired observable in leading and next-to-leading $1 / N_{c}$-order. The same parameter choice as in fig. 2 (i.e. $e=4.25, \gamma=0$ for $\mu=m_{\rho}$ ) leads to the following results: (the first number for a given observable is the classical $\mathcal{O}\left(N_{c}\right)$ result; the second number is the $\mathcal{O}(1)$ one-loop correction; standard experimental values are given in brackets)

$$
\begin{aligned}
& \text { baryon mass: } M=1628-682=946 \quad(939)[\mathrm{MeV}] \\
& \pi \text {-N sigma term: } \sigma=54-21=33 \quad(45)[\mathrm{MeV}] \\
& \text { isovector magn. moment: } \mu_{V}=1.62+0.62=2.24 \quad(2.35)
\end{aligned}
$$$$
\text { isovector magn. square radius: }\left\langle r_{M}^{2}>_{V}=0.86-0.14=0.72 \quad(0.73)\left[\mathrm{fm}^{2}\right]\right.
$$ 
These numbers, quoted from Meier and Walliser [15], allow to conclude that, first of all, the quantum corrections to the classical results are not small. In fact, although we expect them as $1 / N_{c}$ corrections, their magnitude in general exceeds the $30 \%$ estimate (depending on the chosen scale). Still, cum grano salis, they seem to confirm the applicability of the $1 / N_{c}$-expansion, and the fact that they do improve the general agreement with experimental baryonic properties, supports our choice of an effective chiralorder-four lagrangian.

The corresponding consideration for the axial coupling constant $g_{A}$ shows, however, that for certain observables the $1 / N_{c}$-expansion can be more problematic than the numbers given above may suggest. For the same parameter choice the classical $\mathcal{O}\left(N_{c}\right)$ result for $g_{A}$ at $\mu=m_{\rho}$ is $g_{A}=0.90$. At first sight, this appears quite in line with our expectation of a $30 \% \mathcal{O}(1)$ correction to get close to the experimental value of 1.25 . However, the constraint imposed on $g_{A}$ by the Adler-Weisberger relation

$$
g_{A}^{2}=1+\delta, \quad \delta>0,
$$

requires that $g_{A}^{2}$ must contain $\mathcal{O}\left(N_{c}^{0}\right)$ contributions with a numerical magnitude of about 1 , while $g_{A}^{2}$ which is of $\mathcal{O}\left(N_{c}^{2}\right)$ has a numerical value of about 1.5. This shows that in order to reproduce the experimentally observed number for $g_{A}$ in a $1 / N_{c}$ expansion it must be calculated at least to $\mathcal{O}\left(1 / N_{c}\right)$ which is two orders down from the leading $\mathcal{O}\left(N_{c}\right)$, and the resulting corrections to $g_{A}^{2}$ (or to $\delta$ ) will be of the same order of magnitude as the leading term. So, if the tree approximation would happen to be close to the experimental result, one could even expect the one-loop corrections to take it away from this fortunate value to make room for the necessarily large two-loop contributions. And this is what happens with the above choice of parameters:

axial coupling constant: $g_{A}=0.90-0.25=0.65 \quad(1.25)$

The origin of this difficulty is located in the current algebra, which requires the commutator of two axial currents (which both are of $\mathcal{O}\left(N_{c}\right)$ ) to be equal to a vector current (which is of $\mathcal{O}(1)$ ), and it is the nucleon matrix element of this vector current which is responsible for the 1 on the right-hand side of the Adler-Weisberger relation [16]. So, perhaps we should not be too disappointed that the long-standing trouble with $g_{A}$ in soliton models still cannot be resolved at the present level.

\section{RELATIVISTIC FORM FACTORS IN SOLITON MODELS}

A decisive advantage of the soliton concept as compared to all other models where pointlike fermion fields are coupled to mesons or gauge fields is the fact that already in leading classical approximation the spatial structure of the baryon as an extended object is obtained from the underlying effective action. Therefore all types of form factors can readily be extracted from the model itself and precise measurements of their $Q^{2}$-dependence present a severe test for the resulting spatial profiles. Specifically, for electro-magnetic form factors (e.m.FF) new SLAC data [17, 18] pose a challenge for the relativistically corrected FFs of chiral soliton models. And in the few $(\mathrm{GeV} / \mathrm{c})^{2}$ region we expect a wealth of precise data from the new generation of electron accelerators. In this region of momentum transfer it is important to compare data with relativistically corrected FFs.

The implementation of relativistic corrections is fairly easy for solitonic nucleons due to the Lorentz covariance of the underlying field equations. The corrections reflect the Lorentz boost from the soliton rest frame to the Breit frame, in which the soliton moves with velocity $v$ which satisfies

$$
\gamma^{2}=\left(1-v^{2}\right)^{-1}=1+\frac{Q^{2}}{(2 M)^{2}}
$$

for momentum transfer $Q^{2}\left(Q^{2}>0\right.$ in the spacelike region) and soliton mass $M$. The classical result for the magnetic FF is [19] 
where $G^{n r}$ is the nonrelativistic FF evaluated in the soliton restframe. The electric FF $G_{E}$ does not contain the factor $\gamma^{-2}$ on the right-hand side [19]:

$$
G_{E}\left(Q^{2}\right)=G_{E}^{n r}\left(\gamma^{-2} Q^{2}\right)
$$

(this is in contrast to bag models [20] where the wave functions of the spectator quarks supply the factor $\gamma^{-2}$ also for $G_{E}$.)

According to the derivation of $(17,18)$ within tree approximation of the soliton model the kinematical mass $M$ in (16) is the classical soliton mass $M_{S}$, although ideally, of course, $M$ should coincide with the physical nucleon mass $M_{N}$. From $(17,18)$ the asymptotic limit of $G\left(Q^{2}\right)$ for $Q^{2} \rightarrow \infty$ is determined by $G^{n r}\left(4 M^{2}\right)$. For commonly used chiral lagrangians the first zeros of the nonrelativistic FFs occur at masses $M_{0}$

$$
G^{n r}\left(4 M_{0}^{2}\right)=0
$$

which are rather close to the nucleon mass, with $M_{0}<M_{N}$ for $G_{E}^{n r}$ and $M_{0}>M_{N}$ for $G_{M}^{n r}$. This implies that the asymptotic behaviour of $Q^{4} G\left(Q^{2}\right)$ is very sensitive to the precise value of $M$ used in (16-18):

$$
\lim _{Q^{2} \rightarrow \infty} Q^{4} G\left(Q^{2}\right)= \pm \infty \quad \text { for } \quad M \lessgtr M_{0}
$$

The actual values of $M_{0}$ for which $G^{n r}\left(4 M_{0}^{2}\right)$ vanishes, depend on the choice of the parameters in the effective lagrangian; furthermore both, $M_{S}$ and $G^{n r}$ are subject to quantum corrections. It is therefore unrealistic to expect reliable predictions from the model itself for the high- $Q^{2}$ behaviour of $Q^{4} G\left(Q^{2}\right)$.

However, this ambiguity in the high- $Q^{2}$ behaviour of $Q^{4} G\left(Q^{2}\right)$ can be used to impose superconvergence $\left(Q^{2} G_{M}\left(Q^{2}\right) \rightarrow 0\right.$ for $\left.Q^{2} \rightarrow \infty\right)$ on $G_{M}\left(Q^{2}\right)$ by choosing $M=M_{0}$ in $(16,17)$, or, to put it more generally, to check the functional form of (17) against the experimentally observed behaviour of $Q^{4} G\left(Q^{2}\right)$ for large $Q^{2}$ by choosing $M$ as an adjustable parameter. Inclusion of other terms in the lagrangian $\mathcal{L}$, quantum corrections, additional degrees of freedom, may affect the position of zeros in different FFs in different ways. In tree approximation, for a specific choice of $\mathcal{L}$, we therefore should not expect $M$ to be necessarily the same for different formfactors. Due to the lack of the factor $\gamma^{-2}$ on the right hand side in (18), superconvergence cannot be imposed on $G_{E}$ by any choice of $M$.

The low- $Q^{2}$ behaviour is not strongly affected by these variations in $M$, although due to the factor $\gamma^{-2}$ in front of $G_{M}^{n r}$ in (17), even the magnetic radius receives a small contribution from finite values of $M$.

\section{ELECTROMAGNETIC FORM FACTORS OF THE NUCLEON}

We have discussed in the preceding sections that the part of the effective pionic action presently established in the mesonic sector is not sufficient for a realistic description of baryons in the soliton sector. A specific set of higher-order terms is conveniently generated through explicit inclusion of vector mesons. The relevant coupling constants need not necessarily be in close agreement with their values in the mesonic sector as long as we consider observables only in tree approximation. Therefore it may be advisable to keep also an additional small fourth-order term of the Skyrme type (12) in order to supply this important coupling with sufficient strength. As a most simple effective lagrangian we therefore choose the minimal model which comprises $\rho$ and $\omega$ mesons together with the pionic field $U$ in chiral covariant way:

$$
\mathcal{L}=\mathcal{L}^{(2)}+\mathcal{L}_{A}^{(4)}+\mathcal{L}^{(\rho)}+\mathcal{L}^{(\omega)}
$$




$$
\mathcal{L}^{(\omega)}=\int\left(-\frac{1}{4} \omega_{\mu \nu} \omega^{\mu \nu}+\frac{m_{\omega}^{2}}{2} \omega_{\mu} \omega^{\mu}+3 g_{\omega} \omega_{\mu} B^{\mu}\right) d^{3} x
$$

topological baryon current $B_{\mu}$

$$
\begin{gathered}
B_{\mu}=\frac{1}{24 \pi^{2}} \epsilon_{\mu \nu \rho \sigma} \operatorname{tr} L^{\nu} L^{\rho} L^{\sigma} \\
l_{\mu}=\xi^{\dagger} \partial_{\mu} \xi, r_{\mu}=\partial_{\mu} \xi \xi^{\dagger} \text { with } \xi=U^{\frac{1}{2}} .
\end{gathered}
$$

With experimental values for $f_{\pi}=93 \mathrm{MeV}$, meson masses $m_{\pi}=138 \mathrm{MeV}, m_{\rho}=770 \mathrm{MeV}, m_{\omega}=783$ $\mathrm{MeV}$, and $g$ fixed by the KSRF relation $g=m_{\rho} /\left(2 \sqrt{2} f_{\pi}\right)=2.925, \mathcal{L}$ still contains $g_{\omega}$ and $e$ as two free parameters. Their influence on the resulting profiles is rather similar: a small value of $g_{\omega}$ can be compensated by a stronger Skyrme term and vice versa. For all the following results we choose $g_{\omega}=4$ and $e=12$.

The contributions of the vector mesons to the electromagnetic currents are defined through the gauge transformations (with local gauge field $\epsilon$ )

$$
\begin{gathered}
\rho^{\mu} \rightarrow e^{i \epsilon Q_{V}} \rho^{\mu} e^{-i \epsilon Q_{V}}+\frac{Q_{V}}{g} \partial^{\mu} \epsilon \\
\omega^{\mu} \rightarrow \omega^{\mu}+\frac{Q_{0}}{g_{0}} \partial^{\mu} \epsilon
\end{gathered}
$$

(with $Q_{0}=1 / 6, Q_{V}=\tau_{3} / 2$ ). Within our $S U(2)$ scheme we can allow $g_{0}$ to differ from $g$ and thus exploit the freedom in the e.m. coupling of the isoscalar $\omega$-mesons.

The nucleon isoscalar FFs are of very simple form which involves only the spatial distribution of the baryon density $B_{0}(r)$ and the ratio $g_{\omega} / g_{0}$ which determines the contribution of the $\omega$-mesons to the isoscalar part of the e.m. current:

$$
\begin{gathered}
G_{E}^{0}\left(Q^{2}\right)=\frac{1}{2} \int d^{3} r j_{0}(Q r)\left(\frac{g_{\omega}}{g_{0}\left(1+Q^{2} / m_{\omega}^{2}\right)}+1-\frac{g_{\omega}}{g_{0}}\right) B_{0}(r) \\
G_{M}^{0}\left(Q^{2}\right)=\frac{M_{N}}{2 \Theta} \int d^{3} r \frac{j_{1}(Q r)}{Q r}\left(\frac{g_{\omega}}{g_{0}\left(1+Q^{2} / m_{\omega}^{2}\right)}+1-\frac{g_{\omega}}{g_{0}}\right) r^{2} B_{0}(r)
\end{gathered}
$$

(The functional form of other electromagnetic FFs in this model is given e.g. in [21.)

Comparison with the Galster parametrization [22] in figs.3 and 4

$$
\begin{gathered}
G_{E}^{0}\left(Q^{2}\right) / G_{D}\left(Q^{2}\right)=\frac{1}{2}\left(1+\frac{0.54 Q^{2}}{1+1.59 Q^{2}}\right), \quad G_{D}\left(Q^{2}\right)=1 /\left(1+Q^{2} / 0.71\right)^{2} \\
G_{M}^{0}\left(Q^{2}\right) / G_{D}\left(Q^{2}\right)=\mu_{p}+\mu_{n}=\text { const. }\left.\right|_{Q^{2}}
\end{gathered}
$$

shows that $g_{\omega} / g_{0}$ is not equal to one (which would imply complete vector dominance for the isoscalar FFs). Instead, the ratio $g_{\omega} / g_{0}$ should be around $\sim 0.55-0.6$. (This holds for a sufficiently wide range of parameters $e$ and $g_{\omega}$ in the lagrangian). In our case both isoscalar FFs agree well with the Galster parametrization below $Q^{2}<1(\mathrm{GeV} / \mathrm{c})^{2}$, both for the same value of $g_{\omega} / g_{0}=0.58$. This choice then also leads to quite satisfactory agreement for the proton electric FF $G_{E}^{p}\left(Q^{2}\right)$ with the data in the region 
The resulting e.m. FFs for the proton (divided through the standard dipole $G_{D}$ ) are shown in figs.5 and 6, plotted against the logarithm of $Q^{2}$. The dashed lines show the results where both, $G_{E}$ and $G_{M} / \mu_{p}$, are calculated for the same value of the kinematic mass $M=M_{N}=0.94 \mathrm{GeV}$ in (16). The rapid decrease of $G_{E} / G_{D}$ above $Q^{2} \sim 1(\mathrm{GeV} / \mathrm{c})^{2}$ which is in apparent conflict with the SLAC data, has its origin in the fact that the value of $M_{0}$ which characterizes the first low-lying zero of $G_{E}^{n r}$ according to (19) appears at $M_{0}=0.72 \mathrm{GeV}<M_{N}$. With the choice $M=M_{N}$ in (16) the zero in $G_{E}$ is pushed up to $Q^{2} \approx 5(\mathrm{GeV} / \mathrm{c})^{2}$ by the boost to the Breit frame in (18). It can be shifted to higher $Q^{2}$ by decreasing $M$ towards $M_{0}$ (the full line in fig. 5 is calculated for $M=0.85 \mathrm{GeV}$ ). For $M=M_{0}$ the zero is, of course, completely removed (i.e. shifted to infinity). Because the rapid decrease of $G_{E} / G_{D}$ is due to a zero in $G_{E}^{n r}$ it cannot be removed by an additional factor $\gamma^{-2}$ in front of $G_{E}^{n r}$ which may appear in bag models.

For $M=M_{N}$ the proton magnetic FF $G_{M}^{p}\left(Q^{2}\right)$ deviates significantly from the standard dipole $G_{D}$ above $Q^{2}>1(\mathrm{GeV} / \mathrm{c})^{2}$, (dashed line in fig.6). Because for the nonrelativistic magnetic FF we find $M_{0}=1.147 \mathrm{GeV}>M_{N}$, the first zero in $G_{M}^{n r}$ is completely removed by the boost to the Breit frame (i.e. it is shifted beyond infinity into the timelike region of $Q^{2}$ ). The full line in fig.6 is calculated with $M=1.138 \mathrm{GeV}$ which is very close to the requirement of superconvergence $\left(M=M_{0}\right)$. Remarkably, with this small change in the kinematic mass it is possible to obtain the impressive agreement with the SLAC data up to $30(\mathrm{GeV} / \mathrm{c})^{2}$.

For the chosen set of $g_{\omega}$ and $e$ the proton magnetic moment is $\mu_{p}=2.90$, and the neutron magnetic moment is $\mu_{n}=-2.49$. The discrepancy of the latter with its experimental value of -1.91 is due to the fact that in soliton models the isoscalar magnetic moment

$$
\mu_{S} / M_{N}=\frac{<r_{B}^{2}>}{3 \Theta}
$$

is closely tied to the inverse soliton moment of inertia $\Theta$, a relation which is two $N_{c}$ orders down from the $\mathcal{O}\left(N_{c}\right)$ isovector magnetic moment. So, similar to the case of $g_{A}$ discussed earlier, we expect decisive improvement only at the two-loop level. Still it is interesting to note that the resulting Foldy term

$$
\frac{3 \mu_{n}}{2 M_{N}^{2}}=-0.165 \mathrm{fm}^{2}
$$

essentially saturates the calculated square radius of the neutron

$$
<r_{E}^{2}>_{n}=-6 d G_{E}^{n}(0) / d Q^{2}=-0.158 \mathrm{fm}^{2}
$$

(the experimental value is $\left\langle r_{E}^{2}>_{n}=-0.114 \pm 0.003 \mathrm{fm}^{2}\right.$ ). Apparently, the soliton model naturally reproduces the experimental observation that the slope of the Dirac form factor of the neutron $d F_{1 n}(0) / d Q^{2}$ is extremely small (experimentally it is $\sim 0.002 \mathrm{fm}^{2}$ ).

Although details will depend on the choice of parameters in the effective lagrangian it is evident from fig.6 that the functional form (17) is able to describe the general pattern of the observed proton magnetic $\mathrm{FF}$ over the whole range of measured $Q^{2}$ values if superconvergence is imposed, without any further "QCD" corrections [23]. This makes it unlikely to find unambiguous signatures from additional shortrange degrees of freedom in the observed form of $G_{M}^{p}$. A similar result was obtained by Höhler [24] in terms of a suitable parametrization of the spectral function.

The electric FF is dominated by a zero in the few $(\mathrm{GeV} / \mathrm{c})^{2}$ region which is difficult to avoid and which appears to be in conflict with the SLAC data. It is an interesting question whether quantum corrections or inclusion of additional degrees of freedom may shift the first zero in the nonrelativistic electric $\mathrm{FF}$ closer to the value $\left(4 M_{N}^{2}\right)$. The scaling relation $G_{M}^{p}\left(Q^{2}\right) / \mu_{p}=G_{E}^{p}\left(Q^{2}\right)$, however, is satisfied with good accuracy up to $Q^{2} \approx 1(\mathrm{GeV} / \mathrm{c})^{2}$ which is quite remarkable for a model where the Besselfunctions $j_{0}$ and $j_{1}$ determine the electric and magnetic FFs, respectively, (which naively implies for the ratio of the radii $\left\langle r_{M}^{2}>/<r_{E}^{2}>\sim 3 / 5\right)$. Clearly, more experimental information on the proton electric $\mathrm{FF}$ in the 


\section{THE STRONG $\pi N N$ FORM FACTOR}

Let me finally make some remarks concerning the strong form factor which governs the $\pi N N$ interaction in soliton models. First it should be stressed, that the analysis of the meson-baryon scattering S-matrix in the soliton sectors of effective meson lagrangians does not require to separately consider meson-baryon form factors: the spatial structure of the interaction is determined by the selfconsistently calculated soliton profiles and automatically taken care of in the scattering equations. This holds, of course, also for the analysis of the baryon-baryon interaction, or for the structure of the deuteron or other nuclei.

It may, however, be desirable to extract meson-baryon form factors from soliton solutions of mesonic actions, to enable a comparison with the (freely invented) form factors typically used in conventional meson-exchange models of the baryon-baryon interaction. A principal difficulty lies in the fact that effective theories allow for arbitrary unitary redefinitions of the interpolating fields which leave the S-matrix unaffected. It is therefore essential to extract the form factors in such a way that they are independent of the specific definition chosen for the interpolating field. This is indeed possible if one takes due care of the local metric associated with a given choice of interpolating field.

These metrical factors have been disregarded in early attempts to relate the strong form factors to the soliton profiles: the procedure followed in Refs. [25,26] is based on the equation of motion (EOM) for a pion field $\vec{\pi}$ coupled to a (fermionic) axial source

$$
\left(\square-m_{\pi}^{2}\right) \pi^{a}(x)=J_{5}^{a}(x) .
$$

Taking matrix elements for nucleon states and using translational invariance leads to

$$
-\left(q^{2}+m_{\pi}^{2}\right)<N\left(p^{\prime}\right)\left|\pi^{a}(0)\right| N(p)>=<N\left(p^{\prime}\right)\left|J_{5}^{a}(0)\right| N(p)>
$$

with $q=p^{\prime}-p$. The matrix element on the right-hand side defines the form factor $G_{\pi N N}$ through

$$
<N\left(p^{\prime}\right)\left|J_{5}^{a}(0)\right| N(p)>=G_{\pi N N}\left(q^{2}\right) \bar{u}\left(p^{\prime}\right) i \gamma_{5} \tau^{a} u(p)
$$

while the matrixelement on the left-hand side to lowest order in $\hbar / N_{c}$ is the Fourier transform of the classical meson field

$$
<N\left(p^{\prime}\right)\left|\pi^{a}(0)\right| N(p)>=\int e^{i q x} \pi_{c l}^{a}(x) d x
$$

Through (36),(37), and (38) the $\pi N N$ form factor thus is expressed in terms of the classical solution for the chiral field. It implies that in an EOM for the fluctuating pion field derived from any chiral effective action (conveniently formulated in terms of a unitary matrix field $U=\sigma+i \vec{\tau} \cdot \vec{\pi}$ )

$$
\left(\square-m_{\pi}^{2}\right) \pi^{a}(x)=J_{5}^{a}[U(x)]
$$

the matrixelements of the functional $J_{5}^{a}[U(x)]$ in baryonic configurations may be identified with the corresponding fermionic matrix elements of $J_{5}^{a}(x)$.

It should be noted, however, that the EOM derived from some effective meson action is not immediately obtained in the form (39), because the kinetic part will generally contain a local metric. Only after a field redefinition to absorb this metric into the chiral field the correspondingly transformed source function can be compared with the fermionic matrix elements and the form factor. Evidently, this metric can only be identified from the time-derivative part of the action, because any deviation of the spatial part from the required structure $\nabla^{2} \pi^{a}$ could be absorbed into the source function $J_{5}^{a}[U(x)]$ without a redefinition of the field. 


$$
\mathcal{T}=-\frac{f_{\pi}^{2}}{2} \int L_{a}^{0} M_{a b} L_{b}^{0} d^{3} x
$$

This also holds for effective theories which contain more than two time derivatives in their chiral action, because $\mathcal{T}$ is obtained by expanding the lagrangian to second order in the fluctuations. In the Skyrme model and related models the classical field configuration $\pi_{c l}^{a}(x)$ which characterizes the baryon is the hedgehog $U_{0}=\exp (i \vec{\tau} \cdot \hat{x} F(r))$ with chiral profile $F(r)$, rotating in isospace. For solitons of this type the only isovector which can appear in the metric tensor $M_{a b}$ is the pion field itself, $(\vec{\pi}=|\vec{\pi}| \hat{\pi})$, therefore $M_{a b}$ has to be of the form

$$
M_{a b}=M_{L} \hat{\pi}_{a} \hat{\pi}_{b}+M_{T}\left(\delta_{a b}-\hat{\pi}_{a} \hat{\pi}_{b}\right)
$$

with longitudinal and transverse metrical factors $M_{L}$ and $M_{T}$ depending on $\sigma$ and $|\vec{\pi}|$. The metric in (40) can be removed from the kinetic energy by redefining

$$
\tilde{L}_{a}^{0}=M_{a b}^{1 / 2} L_{b}^{0}
$$

For the hedgehog soliton $\vec{\pi}$ rotating in isospace with angular velocity $\vec{\Omega}$ the time derivative $\dot{\vec{\pi}}$ is purely transverse and we have

$$
-\tilde{L}_{a}^{0} \tilde{L}_{a}^{0}=\dot{\tilde{\pi}}_{a} \dot{\tilde{\pi}}_{a}
$$

with redefined field $\tilde{\pi}_{a}=\sqrt{M_{T}} \pi_{a}$.

Combining now eqs.(36-38) with $\pi_{c l}^{a}(x)$ replaced by $\sqrt{M_{T}} \pi_{c l}^{a}$ the $\pi N N$ form factor in the Breit frame then is obtained as

$$
G_{\pi N N}=\frac{8 \pi}{3} \frac{M_{N} f_{\pi}}{Q}\left(Q^{2}+m_{\pi}^{2}\right) \int_{0}^{\infty} d r r^{2} j_{1}(Q r) \sqrt{M_{T}(r)} \sin F(r)
$$

where $M_{T}(r)$ derives from the effective lagrangian used to determine the chiral profile $F(r)$. As a typical example, we consider the standard Skyrme lagrangian. It leads to the transverse metric to be used in $(44)$

$$
M_{T}(r)=1+\frac{1}{e^{2} f_{\pi}^{2}}\left(F^{2}+\frac{\sin ^{2} F}{r^{2}}\right)
$$

This is the form factor which implicitly underlies the successful application of the soliton model to $\pi N$ elastic scattering [14,27,28]. In fig.7 we compare it with conventional monopole form factors $G_{0}\left(Q^{2}\right)=$ $\left(\Lambda^{2}-m_{\pi}^{2}\right) /\left(\Lambda^{2}+Q^{2}\right)$ with $\Lambda=0.8$ and $1.7 \mathrm{GeV}$. The contribution of the Skyrme term (with $e=4.25$ ) to the pionic metric causes a qualitative change in the low- $Q^{2}$ behaviour of the formfactor: the slope near $Q^{2}=0$ is very small and the curvature is negative. This means that for small $Q^{2}$ the effective $\pi N N$ coupling strength stays much closer to its value at $Q^{2}=-m_{\pi}^{2}$ than for comparable monopole form factors. It is this feature of the Skyrme model form factor which also improves agreement of the calculated P33 phase shifts in $\pi N$ scattering with the data in the $\Delta$-resonance region [29]. If the metric factor in (44) is omitted (i.e. (45) replaced by $M_{T}(r)=1$ ) then the resulting $\mathrm{FF}$ is very close to a soft monopole FF with $\Lambda \sim 0.8 \mathrm{GeV}$, as originally observed in [25,26]. Soft $(\Lambda \sim 0.8 \mathrm{GeV})$ monopole FFs fail in the NN system, since they cut out too much of the tensor force provided by the pion: the deuteron quadrupole moment and asymptotic D/S state ratio and the $\epsilon_{1}$ mixing parameter of NN scattering (which all depend crucially on the nuclear tensor force) come out too small [30]. The very hard behaviour of the Skyrme model FF for small $Q^{2}$ therefore proves very helpful in standard OBE calculations. On the other hand the very soft behaviour for $Q^{2}>50 m_{\pi}^{2}$ cuts off higher momenta much more efficiently than typical hard monopole formfactors. Table 1 shows some results obtained by Machleidt [32] for deuteron properties and low-energy n-p scattering with the standard monopole 
Tab. 1. Deuteron and low energy scattering parameters as predicted by the two OBE potential models discussed in the text. Column 'Experiment' gives the empirical values.

\section{Bonn-B Skyrme FF Experiment ${ }^{a}$}

$\begin{array}{llll}\text { Deuteron: } & & & \\ \text { Binding energy }-\epsilon_{d}(\mathrm{MeV}) & 2.2246 & 2.22454 & 2.224575(9) \\ \text { D-state probability } P_{D}(\%) & 4.99 & 4.71 & - \\ \text { Quadrupole moment } Q_{d}\left(\mathrm{fm}^{2}\right) & 0.278^{b} & 0.274^{b} & 0.2860(15) \\ \text { Magnetic moment } \mu_{d}\left(\mu_{N}\right) & 0.8514^{b} & & 0.857406(1) \\ \text { Asymptotic S-state } A_{S}\left(\mathrm{fm}^{-1 / 2}\right) & 0.8860 & 0.8876 & 0.8846(8) \\ \text { Asymptotic D/S-state } D / S & 0.0264 & 0.0257 & 0.0256(4) \\ \text { Root-mean-square radius } r_{d}(\mathrm{fm}) & 1.9688 & & 1.968(5)\end{array}$

Neutron-proton low-energy scattering: (scattering lenght $a$, effective range $r$ )

\begin{tabular}{|c|c|c|c|}
\hline$S_{0}: a_{n p}(\mathrm{fm})$ & -23.75 & -23.75 & $-23.748(10)$ \\
\hline$r_{n p}(\mathrm{fm})$ & 2.71 & 2.73 & $2.75(5)$ \\
\hline${ }^{3} S_{1}: a_{t}(\mathrm{fm})$ & 5.424 & 5.434 & $5.419(7)$ \\
\hline$r_{t}=\rho(0,0)(\mathrm{fm})$ & 1.761 & 1.776 & $1.754(8)$ \\
\hline
\end{tabular}

\footnotetext{
${ }^{a}$ The figures in parentheses after the experimental values give the one-standard-deviation uncertainties in the last digits. A comprehensive list of references for the experimental values is given in Table 4.2 (p. 227) of Ref. [31].

${ }^{b}$ The meson-exchange current contributions to the moments are not included in the predictions.
}

\section{CONCLUSION}

First attempts to relate the soliton description of baryons to the established parts of the low-energy effective action of ChPT have shown that for application in the soliton sector more knowledge about the higher chiral orders in $\mathcal{L}$ is necessary. However, as a model with 'effective' low-energy coupling constants a truncated form of $\mathcal{L}$ proves very useful for studying the next-to-leading-order terms in the $1 / N_{c}$ expansion. It turns out that they in fact constitute sizable contributions to physical observables and remarkably improve the overall quality of the soliton picture. For some quantities, especially for $g_{A}$, we even have to expect large next-to-next-to-leading-order corrections. It seems not to be possible to consistently absorb these quantum corrections into a suitable choice of effective coupling constants for the tree approximation even with inclusion of vector mesons.

We have presented a detailed comparison of e.m. FFs (evaluated in tree approximation with relativistic corrections) with recent data. It shows the capability of the soliton model to describe the peculiar features of the FFs over a large range of $Q^{2}$, depending on the precise position of the first zeros in the nonrelativistic FFs. In the model we have used here the electric proton FF is characterized by a zero in the few $(\mathrm{GeV} / \mathrm{c})^{2}$ region which we find difficult to avoid. It would be interesting to see how this feature is affected by inclusion of further mesonic degrees of freedom, or by loop corrections. But in any case, the quality of agreement which seems possible within these models will make it difficult to see clear signatures from the quark structure of nucleons in the presently available data of electro-magnetic form factors. 


\section{REFERENCES}

[1] S. Weinberg, Physica 96A (1979) 327.

[2] J. Gasser and H. Leutwyler, Ann. Phys.(NY) 158 (1984) 142; Nucl. Phys. B250 (1985) 465.

[3] T.H.R. Skyrme, Proc. Roy. Soc. Ser. A260 (1961) 127.

[4] E. Witten, Nucl. Phys. B223 (1983) 422, 433.

[5] U.G. Meissner, N. Kaiser and W. Weise, Nucl. Phys. A466 (1987) 685.

[6] P. Jain, R. Johnson, U.G. Meissner, N.W. Park, and J. Schechter, Phys. Rev. D37 (1988) 3252.

[7] U.G. Meissner, N. Kaiser, H. Weigel, and J. Schechter, Phys. Rev. D39 (1989) 1956.

[8] B. Schwesinger, H.Weigel, G.Holzwarth, and A. Hayashi, Phys. Reports 173 (1989) 173; and references therein.

[9] B. Moussallam, Ann. Phys.(NY) 225 (1993) 264;

B. Moussallam and D. Kalafatis, Phys. Lett. B272 (1991) 196;

B. Moussallam, in Proc. Int. Workshop "Baryons as Skyrme solitons", Siegen Sept. 1992, World Scientific P.C., Singapore 1993, 269-278.

[10] G. Holzwarth, Nucl. Phys. A572 (1994) 69.

[11] G. Holzwarth and H. Walliser, Nucl. Phys. A587 (1995) 721.

[12] R. Rajaraman: "Solitons and Instantons", North Holland, Amsterdam 1982.

[13] I. Zahed, A. Wirzba and U.G. Meissner, Phys. Rev. D33 (1986) 830;

A. Dobado and J. Terron, Phys. Lett. B247 (1990) 581.

[14] H. Walliser and G. Eckart, Nucl. Phys. A429 (1984) 514;

A. Hayashi, G. Eckart, G. Holzwarth, and H. Walliser, Phys. Lett. 147B (1984) 5;

M.P. Mattis and M. Karliner, Phys. Rev. D31 (1985) 2833;

M. Karliner and M.P. Mattis, Phys. Rev. D34 (1986) 1991;

G. Eckart, A. Hayashi, and G. Holzwarth, Nucl. Phys. A448 (1986) 732.

[15] F. Meier and H. Walliser, Siegen preprint 1995, to be published.

[16] D.O. Riska and M. Kirchbach, Nuovo Cim. 104A (1991) 1837.

[17] A.F. Sill et al., Phys. Rev. D48 (1993) 29.

[18] L. Andivahis et al., Phys. Rev. D50 (1994) 5491;

R.C. Walker et al., Phys. Rev. D49 (1994) 5671.

[19] Xiangdong Ji, Phys. Lett. B254 (1991) 456.

[20] M.V.Barnhill, Phys. Rev. D20 (1979) 723;

M. Betz and R. Goldflam, Phys. Rev. D28 (1983) 2848;

X.M. Wang and P.C. Yin, Phys. Lett. B140 (1984) 249;

C.J. Benesh and G.A. Miller, Phys. Rev. D36 (1987) 1344.

[21] F. Meier, in: Baryons as Skyrme solitons, ed. G. Holzwarth (World Scientific, Singapore 1993) p.159.

[22] S. Galster et al., Nucl. Phys. B32 (1971) 221.

[23] See refs. ${ }^{4-8,30-51}$ in A.F. Sill et al. [17].

[24] G. Höhler et al., Nucl. Phys. B114 (1976) 505;

G. Höhler: $\pi N$ Newsletter No. 9 (1993) 108, ISSN 0942-4148.

[25] T. D. Cohen, Phys. Rev. D34, 2187 (1986).

[26] N. Kaiser, U. G. Meissner, W. Weise, Phys. Lett. B198, 319 (1987);

N. Kaiser, U. Vogl, W. Weise, U. G. Meissner, Nucl. Phys. A484, 593 (1988).

[27] H. Walliser, Nucl. Phys. A524 (1991) 706;

D. Masak, H. Walliser, and G. Holzwarth, Nucl. Phys. A536 (1992) 583.

[28] G. Holzwarth, G. Pari, and B.K. Jennings, Nucl. Phys. A515 (1990) 665

[29] G.Holzwarth, in: 'Baryons as Skyrme solitons', ed. G. Holzwarth (World Scientific, Singapore 1993) p.279.

[30] See Fig. 4.6 (p. 233) and related text of Ref. 31.

[31] R. Machleidt, Adv. Nucl. Phys. 19, 189 (1989). 
Fig. 2. The scale dependence of the baryon mass $M(\mu)$ in tree + one-loop approximation (full line). The dashed line shows the soliton mass $M_{S}(\mu)$ which is implicitly scale dependent through the renormalized coupling constants $e(\mu)$ and $\gamma(\mu)$ in the lagrangian (12-14). (From [15]).

Fig. 3. The isoscalar electric FF (divided by the dipole FF $G_{D}\left(Q^{2}\right)$ ) for three different values of $g_{\omega} / g_{0}=1$ (lowest curve), 0.58 (middle curve), 0 (upper curve). The dashed line is the Galster parametrization of eq.(30).

Fig. 4. The isoscalar magnetic FF (divided by the dipole FF $G_{D}\left(Q^{2}\right)$ ) for $g_{\omega} / g_{0}=1,0.58,0$ (as in fig. 3). The dashed line is the constant $\mu_{0}=\mu_{p}+\mu_{n}$.

Fig. 5. The electric FF of the proton $G_{E}^{p} / G_{D}$ (divided by the dipole $G_{D}$ ) for the kinematic mass $M=0.94$ $\mathrm{GeV}$ (dashed line) and $M=0.85 \mathrm{GeV}$ (full line). The data points are from the compilation of refs. [24] (open circles) and [18] (triangles).

Fig. 6. The magnetic FF of the proton $G_{M}^{p} / G_{D}$ (divided by the dipole $G_{D}$ ) for the kinematic mass $M=0.94$ $\mathrm{GeV}$ (dashed line) and $M=1.138 \mathrm{GeV}$ (full line). The data points are from the compilation of refs. 24] (open circles), [17] (dots), and [18] (triangles).

Fig. 7. The normalized $\pi N N$ formfactor (44) for the Skyrme model with $e=4.25$ (full line). The dotted and dashed lines show the conventional monopole form factors for $\Lambda=1.7 \mathrm{GeV}$ and $\Lambda=0.8 \mathrm{GeV}$, respectively. 


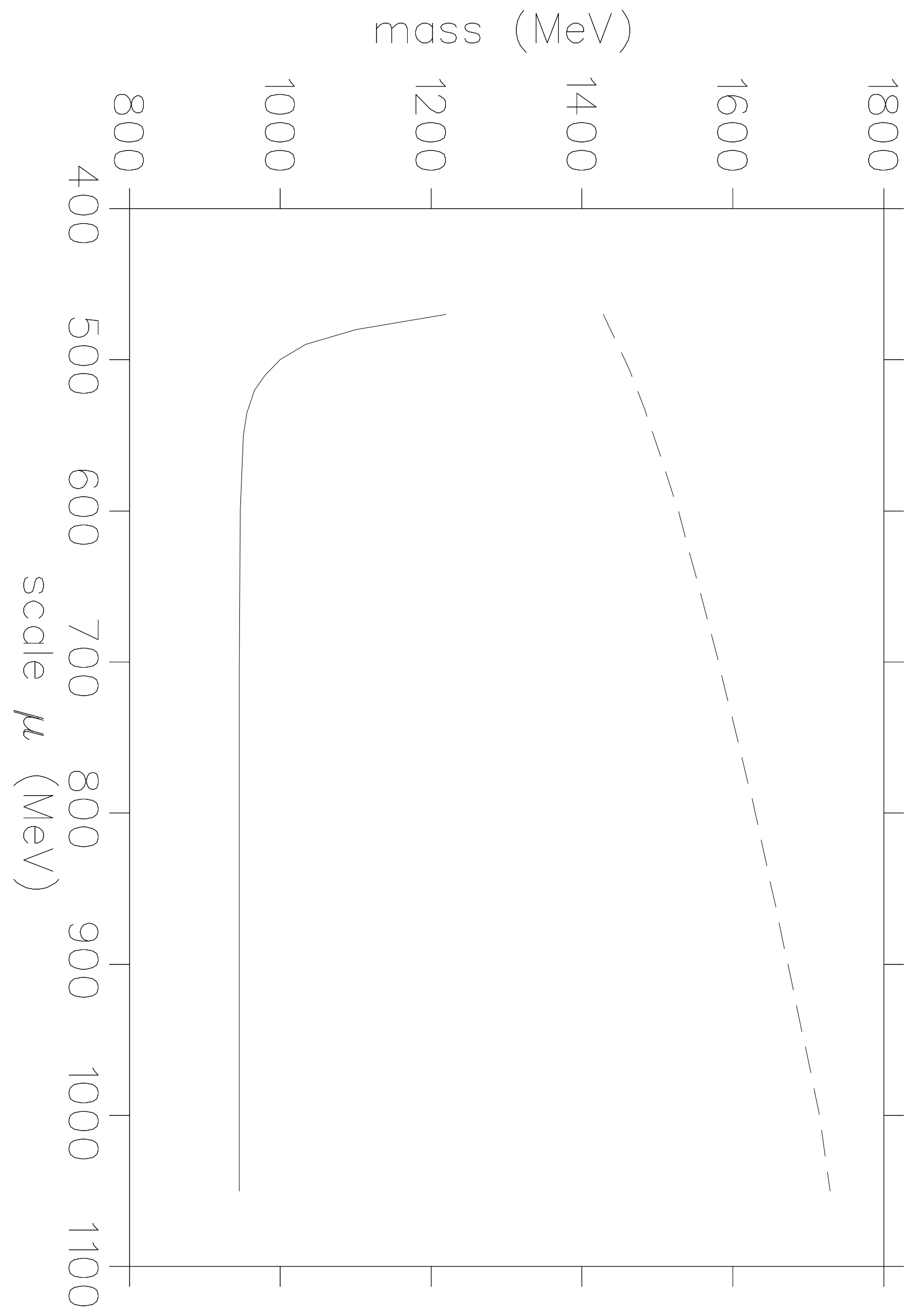




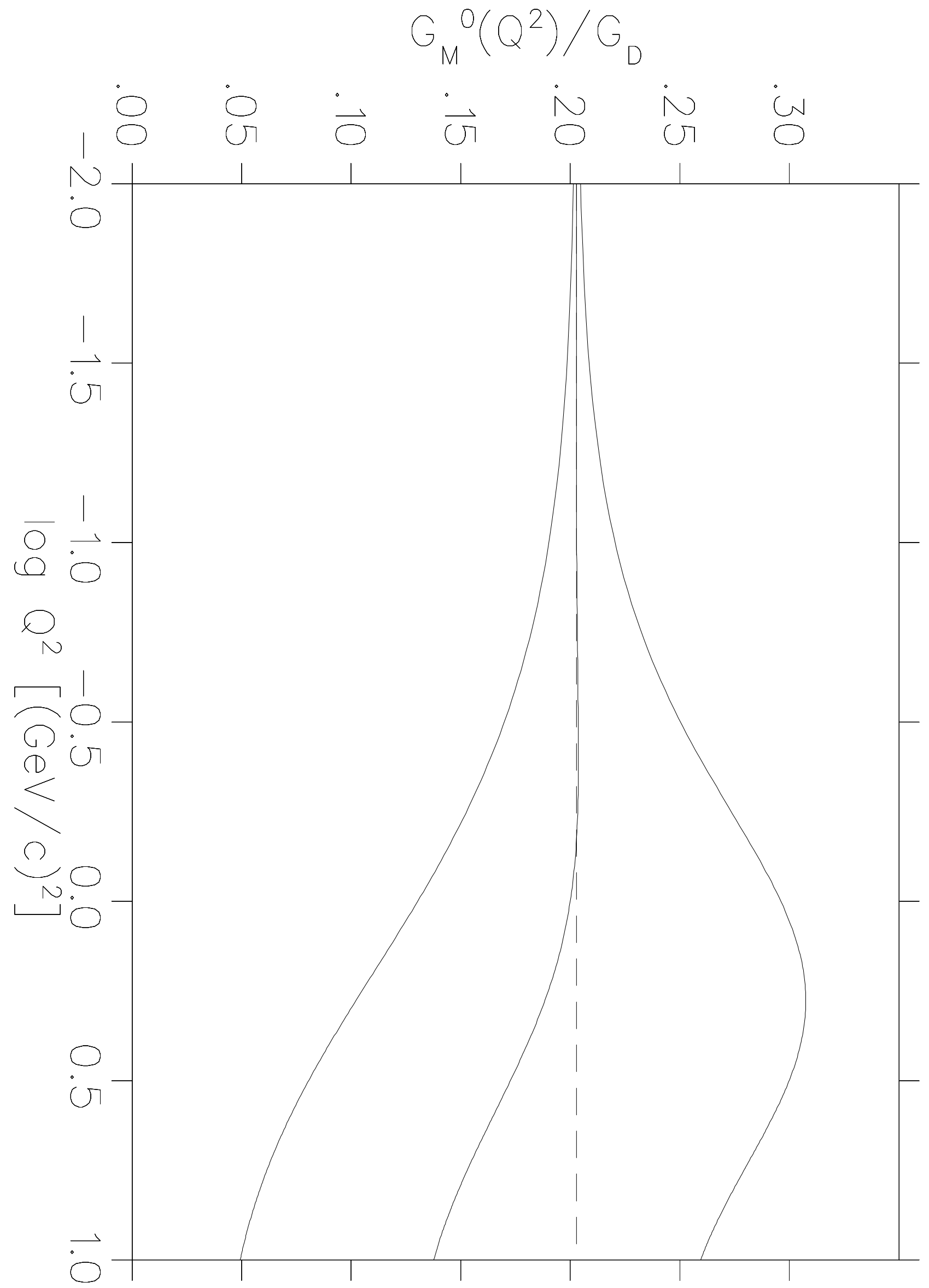




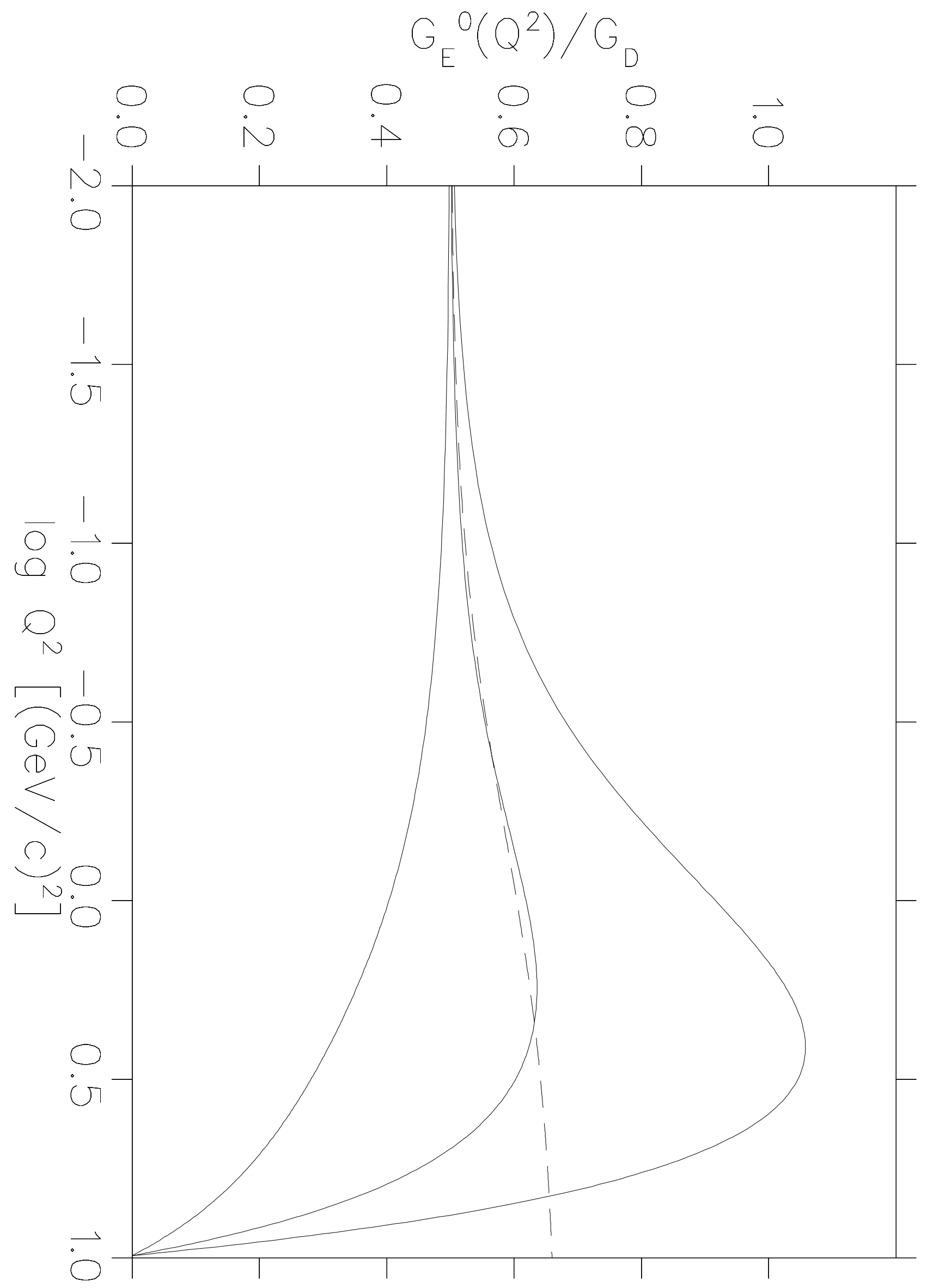




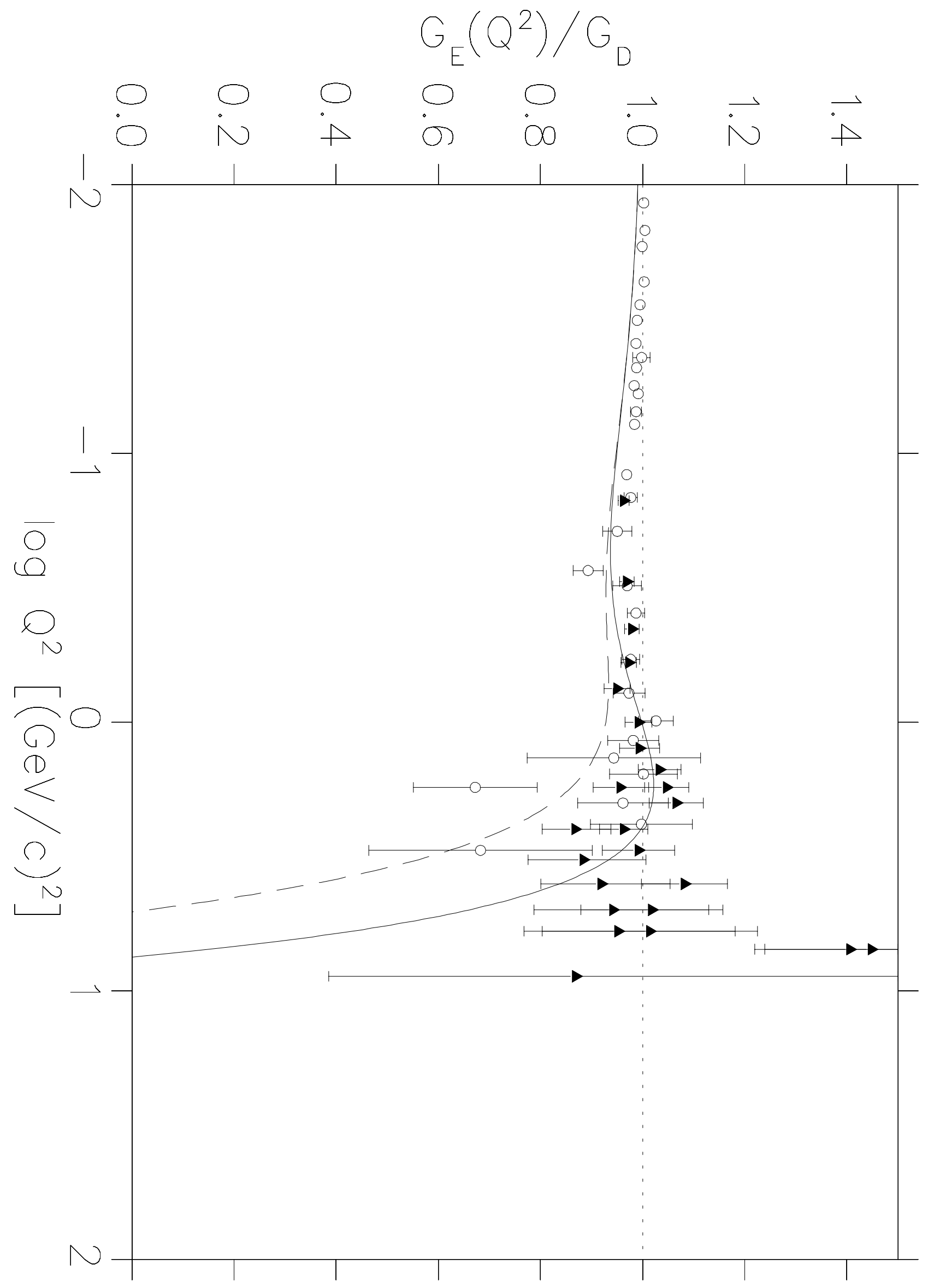




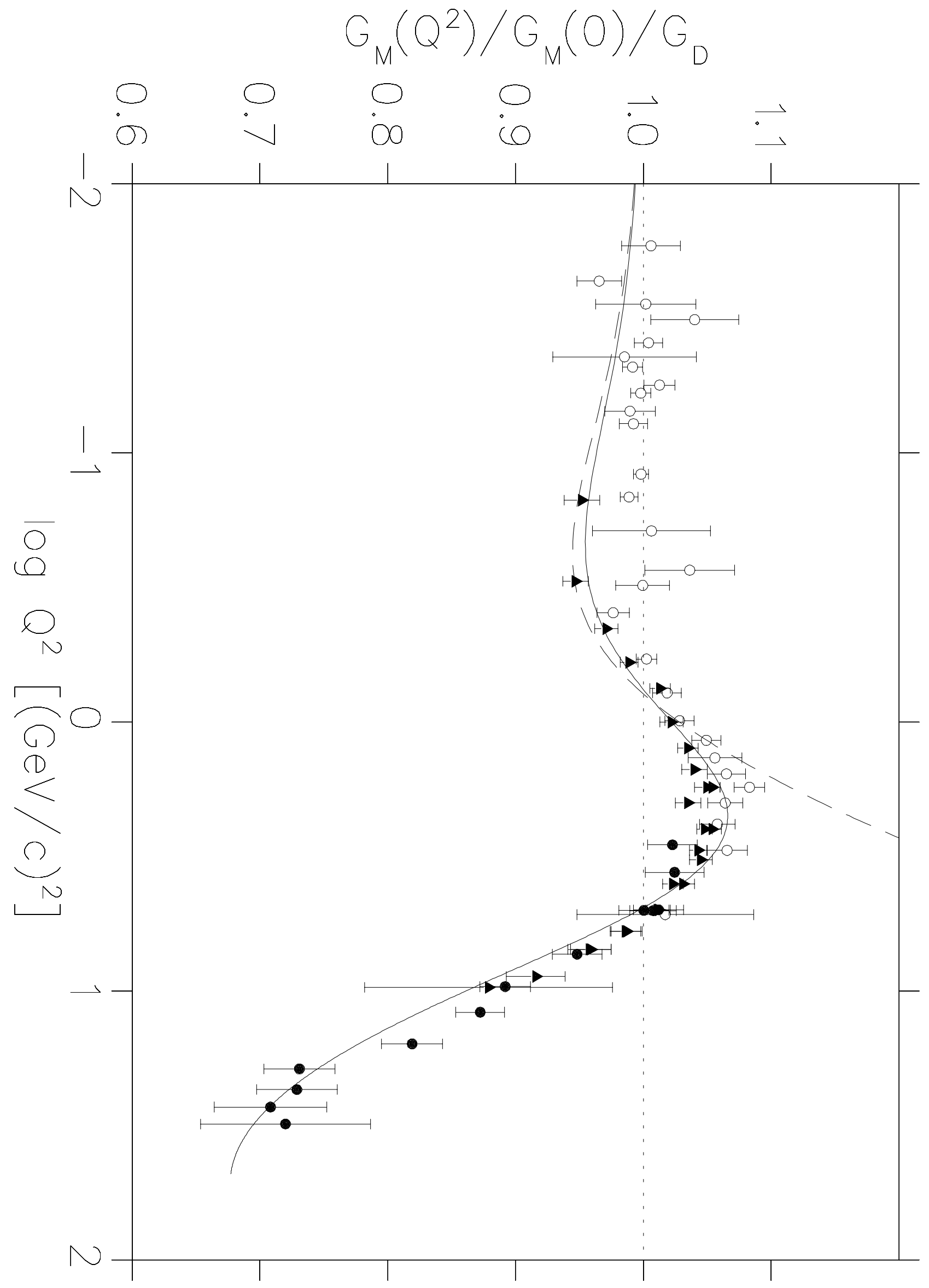




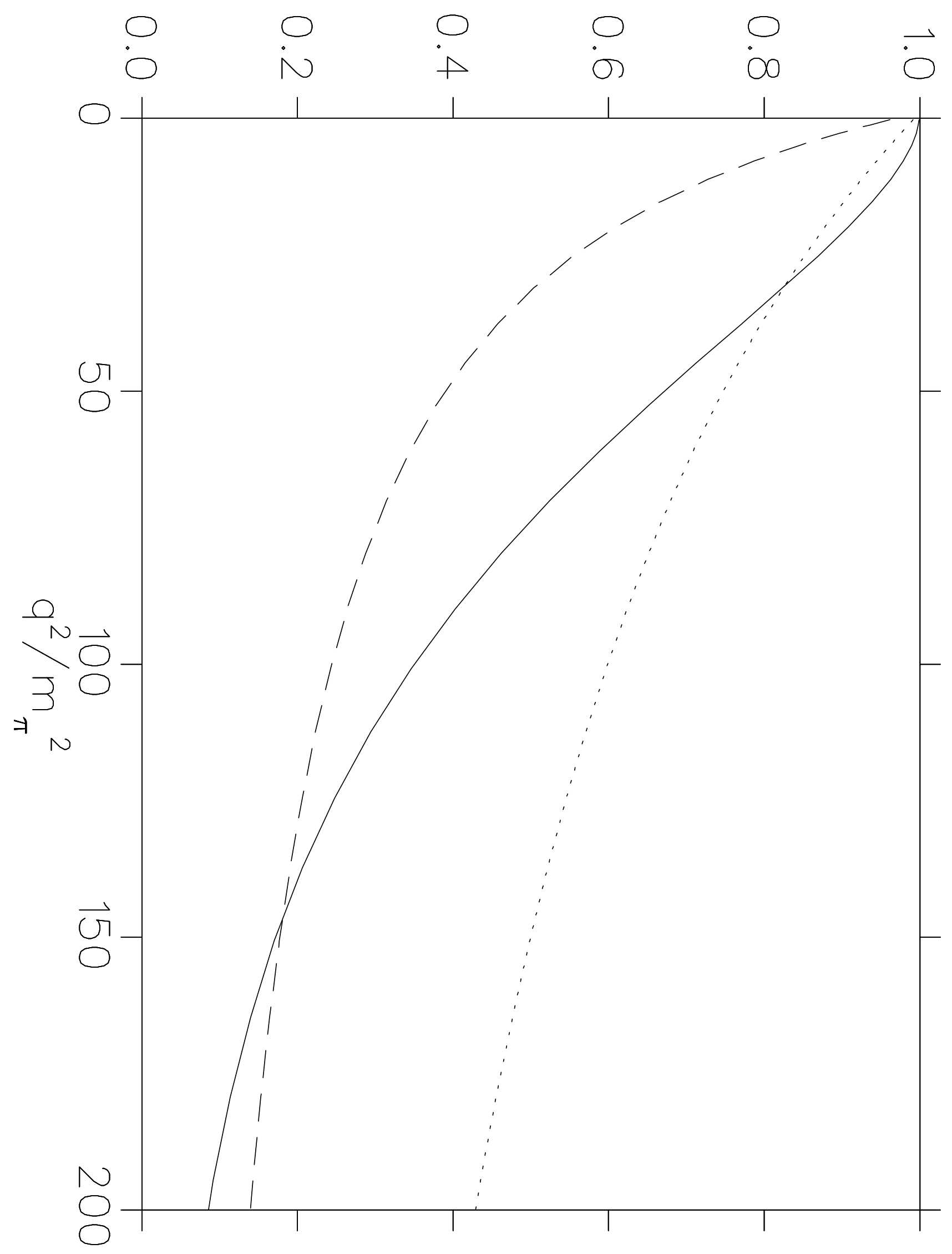

